\title{
Ethos, transformações e permanências
}

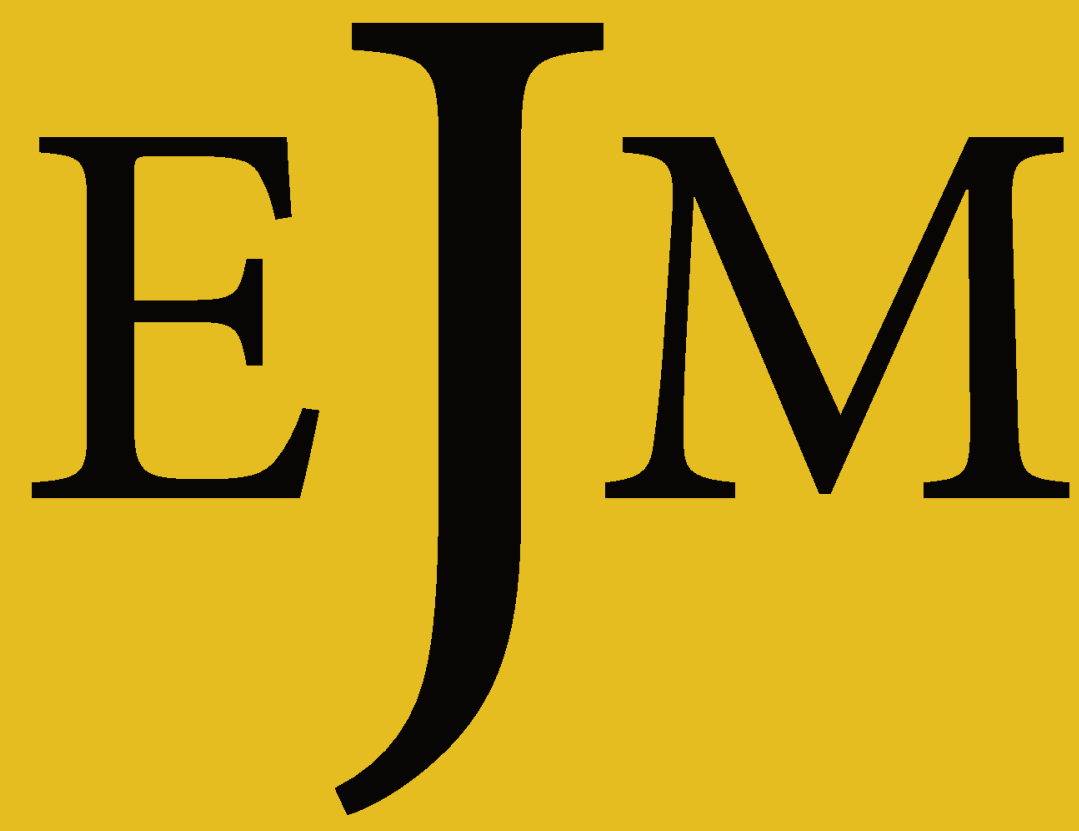

Revista Acadêmica Semestral

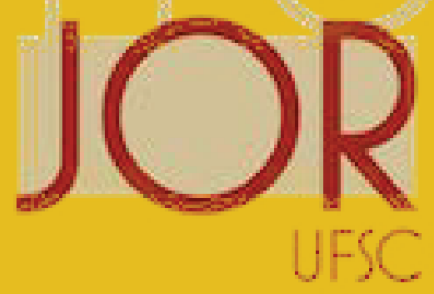

Programa de Pós-Graduação em Jornalismo

Universidade Federal de Santa Catarina

Volume $15 \mathrm{n}^{\circ}$ 1, Janeiro a Junho de 2018 


\section{Universidade Federal de Santa Catarina}

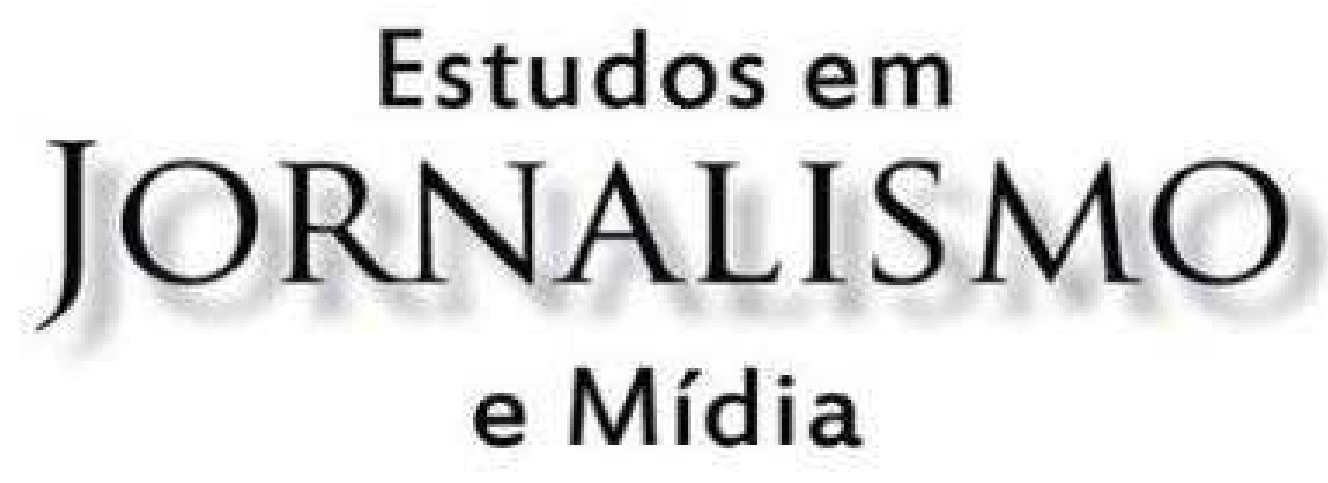

Volume 15 - No 1

Janeiro a Junho de 2018

ISSNe 1984-6924

(c) 198 


\title{
Universidade Federal de Santa Catarina
}

\author{
Reitor \\ Ubaldo Cesar Balthazar \\ Pró-Reitor de Pesquisa \\ Sebastião Roberto Soares \\ Pró-Reitor de Pós-Graduação \\ Hugo Moreira Soares \\ Centro de Comunicação e Expressão \\ Diretor \\ Arnoldo Debatin Neto \\ Departamento de Jornalismo \\ Chefe \\ Maria José Baldessar \\ Programa de Pós-Graduação em Jornalismo \\ Coordenadora \\ Carlida Emerim
}

Estudos em Jornalismo e Mídia - Volume 15, nº1, Janeiro a Junho de 2018

Semestral

Revista Acadêmica Semestral On-line - Programa de Pós-Graduação em Jornalismo da Universidade Federal de Santa Catarina

ISSNe 1984-6924

1. Jornalismo 2. Mídia I.Título

Universidade Federal de Santa Catarina

Programa de Pós-Graduação em Jornalismo

http://www.posjor.ufsc.br

Florianópolis - SC - Brasil

Fone: +55 48 3721-6610

Revista Estudos em Jornalismo e Mídia

http://periodicos.ufsc.br/index.php/jornalismo

E-mail: ejm@contato.ufsc.br 


\section{Estudos em \\ JORNALISMO \\ e Mídia}

Comissão Editorial: Jorge Kanehide Ijuim e Terezinha Silva (editores)

Projeto Gráfico e Capa: Sílvio da Costa Pereira e Ana Carolina Passos

Entrevistador: Laerte Cerqueira

Revisão: Ana Marta Moreira Flores (traduções), Cândida de Oliveira, Criselli Maria Montipó,

Dairan Paul, Edwin Carvalho, Ingrid Pereira de Assis, Janaíne Kronbauer dos Santos, Janara

Nicoletti, Jessica Gustafson, Juliana Gobbi Betti, Karina Woehl de Farias, Marcionize Bavaresco,

Marli Vitali, Rafael Rangel Winch, William Robson Cordeiro Silva.

Editoração e produção: Isabela Martins

Apoio de Produção: Sílvio da Costa Pereira e Equipe do Portal de Periódicos da

UFSC

\section{Conselho Editorial}

Adriana Amado, UNLAM, Argentina

Aglair Bernardo, UFSC, Brasil

Alfredo Vizeu, UFPE, Brasil

Antonio Hohlfeldt, PUC-RS, Brasil

Ana Carolina Escosteguy, PUC-RS, Brasil

Ana Paula Goulart Ribeiro, UFRJ, Brasil

Basilio G. Monteiro, Saint John's University, EUA

Beatriz Becker, UFRJ, Brasil

Carlos Eduardo Franciscato, UFS, Brasil

Claudia Lago, ECA/USP, Brasil

Claudia Mellado Ruiz, Usach, Chile

Christa Berger, Unisinos, Brasil

Dione Oliveira Moura, UnB, Brasil

Dóris Fagundes Haussen, PUC-RS, Brasil

Edson Fernando Dalmonte, UFBA, Brasil

Eduardo Meditsch, UFSC, Brasil

Elias Machado Gonçalves, UFSC, Brasil

Elton Antunes, UFMG, Brasil

Flávio Porcello, UFRGS, Brasil

Francisco José Castilhos Karam, UFSC, Brasil

Gislene Silva, UFSC, Brasil

Heloíza Herscovitz, California State Univ., EUA
Isabel Ferin Cunha, U. Coimbra, Portugal Jacques Mick, UFSC, Brasil

João Canavilhas, UBI, Portugal

Jorge Pedro Sousa, Un. Fernando Pessoa, Portugal Josenildo Luiz Guerra, UFS, Brasil

Juan Carlos Suárez Villegas, Un. Sevilla, Espanha

Kênia Beatriz Ferreira Maia, UFRN, Brasil

Lila Luchessi, UNRN, Argentina

Luiz Gonzaga Motta, UnB-UFSC, Brasil

Manuel Pinto, Universidade do Minho, Portugal

Marcos Palácios, UFBA, Brasil

Marcia Benetti, UFRGS, Brasil

Maria José Baldessar, UFSC, Brasil

Martín Becerra, UBA, Argentina

Nilson Lemos Lage, UFSC, Brasil

Paulo Bernardo Ferreira Vaz, UFMG, Brasil

Rosana Soares, USP, Brasil

Silvio Waisbord, George Washington Univ., EUA

Sonia Virgínia Moreira, UERJ, Brasil

Susana Herrera Damas, Univ. Carlos III, Espanha

Suzana Barbosa, UFBA, Brasil

Victor Gentilli, UFES, Brasil 